\title{
Die Hornhautvaskularisation bei Diemictylus pyrrhogaster.
}

\author{
Von
}

\section{Takamichi Tsusaki.}

(Aus dem Aratomischen Institut der Medizinischen Hochschule zu Keijo).

\author{
Mit ¿ Textfiguren.
}

Unter 'normalen Verhältnissen fehlt es der Hornhaut im allgemeinen an Blutgefässen; die ja die Durchsichtigkeit wesentlich beeinträchtigen würden. Früher glaubte nan, dass die Hornhaut des Menschen im embryonalen Leben von vielen Blutgefässen versorgt werde. Fo war aber ein Kunstprodukt, das infolge der verschiedenen Behandlungen entstanden war. Es können hier aber pathologisch Gefäisse ausgebildet werden. So ist die Hornhaut also ganz gefässlos, mit Ausnahme der äussersten Randbezirke. Durch weitere genanere Untersuchungen wurde aber später festgestellt, dass die Hornhaut bei einigen Tieren auch im ganz normalen Zustand mit Gefässen versehen ist.

Am Rande der Kornea von Huchen findet Berger (1) ein arkadenförmiges Gefässnetz, von welchem jedoch noch einzelne kleine gegen den mittleren Teil der Hornhaut sich begebende Gefässästchen abgehen und eine kurze Strecke weit sich verfolgen lassen. Sonst erscheint an der Hornhautperipherie beim Huchen, nach demselben Autor, ein grosser Teil der aus den Ciliarnerven entstandenen Nerveustämmchen von einem zarten Gefässe begleitet. Eine ähnliche Beobachtung hat Hoyer auch an dènselben Nervenstämmchen des Hechtes gemacht. Berger (1) fügt hinzu, dass die Ciliarnerven bereits in ihrem Verlaufe im suprachorioidalen Lymphraume und zwar im vorderen Teile desselben von kleinen Gefäissen begleitet werden.

Hirsch (4) tritt der Lehre von einem präkornealen fötalen Gefässnetz mit aller Entschiedenheit entgegen. Er injizierte die Gefässe der Schweine von 20.5 bis $155.0 \mathrm{~mm}$, Schafe bis zu $100.0 \mathrm{~mm}$, Kaninchen von 21.0 bis $52.0 \mathrm{~mm}$ Köperlänge und einzelne menschlicher Föten und fand; dass nur eine höchstens $1.0 \mathrm{~mm}$ breite Randzone der Hornhaut stets vaskularisiert. Die Gefässe schlısen 
jedoch nicht mit Randschlingen, sondern mit einem Ringgefïss ab und bestanden in einem Netz von Kapillaren. Die Zahl der zuführenden Arterien beläuft sich durchschnittlich auf fünf, (lie der Venen ist-doppelt so gross.

Motais (9) ist auch derselben Meinung wie Berger(1) und schreibt in Poirier und Charpys Handbuch wie folgt: "Chez quelques poissons osseux, la carpe en particulier, la cornée est vasculaire pendant toute la vie. On attribute ordinairement à la cornée les fins vaisseaux occupant la région du limbe sclérocornéen sur une largeur de $1.0 \mathrm{~mm}$ environ et dont la convexité est tournée vers la cornée. Le sang vient des artères ciliaires antérieures et retourne au plexus veineux épiscléral qui se jette dans les veines ciliaires antérieures. Le réseau profond présente un degré de développement très variable. II est formé ègalement par des anses; celles-ci proviennent des vaisseaux qui accompagnent les nerfs à leur entrée dans la cornée."

Nach Pütter (11) kommen Kapillaren bei Cyprinus carassius fast mitten in der Kornea vor.

Unter den Urodelen wurde das Vorhandensein von Blutgefässen der Hornhaut erst von Rejsek (12), später von Deyl (3) bei Cryptobranchus japonicus nachgewiesen. Sonst haben wir noch diesbezüglich die Arbeit von Lauber(8), die besonders genauere Beschreibung des Auges desselben Tieres gibt. Dabei sagt er : “Eine besondere Eigentümlichkeit der Hornhaut von Cryptobranchus japonicus sind die starken Gefässe, von denen sie durchzogen wird. Sie stellen die Fortsetzung der Gefässe der Cutis dar und sind so dicht angeordnet, dass die von Rejsek gemachte Anyabe, er habe wegen der dichten Hornhautgefässe den Fundus des Auges nicht ophthalmoskopisch sehen können, glaubwürdig erscheint."

Krause (7) fand auch bei drei Exemplaren von Cryptobranchus einen dichten subepithelialen Plexus von Blutgefässen in der Kornea.

Ito (5) und Murata (10) konnten bei Cryptobranchus japonicus auch die Augabe jener Autoren bestätigen.

Das Vorkommen von Blutgefässen in der normalen Hornhaut der Urodelen ist meines Wissens ausser von Rejsek, Deyl, Lauber, Ito und Murata bei Cryptobranchus japonicus, noch bei keinen anderen Urodelen beschrieben worden. $\mathrm{Da}$ es mir interessant schien, zu untersuchen, wie weit diese Gefässe bei den Urodelen verbreitet sind, so habe ich die Hornhaut einiger in Japan einheimischen Urodelen untersucht. Folgende Urodelen kamen in.Betracht : Cryptobranchus 
japonicue, Diemictylus pyrrhogaster, Onychodactylus japonicus, Hynobius nebulosus und Hynobius leechii.

Um die Vaskulariation der Hornhant bei Cryptobranchus zu sehen, genügt einfache makroskopische Untersuchung oder solche mit Lupe, wobei man hier einen zierlichen Gefäsplexus, selbst mit nackten Augen, mit Klarbeit konstatieren kann. Für den histologischen Zweck wurden die Tiere mit chinesischer Tusche ron der Herzkanmer aus injiziert und sofort in 95\% igen Alkohol gelegt. Dann wurde die Hornhant exstirpiert, in dicke oder dünne Schnitte zerlegt, die teils gefärbt, teil: ungefärbt untersucht wurden. Zur Fārbung wurde HämatoxylinEosin angewandt.

Es fand sich bei fünf untersuchten Exemplaren von Cryptobranchus japonicus immer ein dichter, in der Substantia propria cornea befindlicher Plexus von Blutgefissen. Diese Tatsache spficht entschieden dafür, dass das Vorhandensein von-Kapillaren in der Hornhaut hier als ein normaler Zustand zu betrachten ist. Anf Genaueres will ich nicht weiter eingehen, weil es schon von oben genannten Autoren mehrfach untersucht wurde.

Von Onychodactylus, Hynobius nebulosus and Hynobius leechii habe ich nur kurz zu berichten. Unter normalen Verhāltnissen ist die Hornhaut bei diesen Tieren gefässlos, mit Ausnahme der äussersten Randbezirke, woselbst die sogenannten Randschlingennetze sich finden. Sie reichen aber nicht über den Limbu: hinaus. Die Randschlingennetze empfangen ihren arteriellen Zufluss von Aa. ciliares anteriores nnd dann von den Arterien der Bindehaut. Man kann zrei Arten von Netzen nach der Quelle der Gefãse unterscheiden, ein oberflächliches, direkt unter dem Epithel gelegenes und ein tiefes zu den mittleren Schichten der Substantia propria gehörendes Gefässnetz. Die Netze gehen schlingenförmig in die $\mathrm{V} v$. ciliares anteriores über, die mit den gleichnamigen Arterien lanfen. Während das oberflächliche Netz aus einem kontinuierlichen Netzwerk feinster Gefässchen besteht, strahlen die tiefen Gefasse in gewissen Abständen radiär in die Hornhaut ein.

Jetzt kommt Diemictylus pyrrhogaster in Betracht. Mein Material besteht aus 25 Exemplaren. Bei diesem Tier verhält sich die Sache in den meisten Fällen ganz eben so wie bei vorigen drei Arten. Manchmal kommen aber die Gefasse in der Mitte der Hornhaut vor, so das ich dies in einem Fall mit voller Sicherheit konstatieren konnte. Daher ist unter den Crodelen, mit Ausnahne von Crypto- 
branchus japonicus, das Kornealgefägs bei diesem Tiere allein festgestellt. Es fragt sich jedoch, ob wir es vielleicht mit einem pathologischen Befunde gu tun haben. Dies scheint mir nicht der Fall za sein, da am betreffenden Auge keinerlei entzündliche Veränderungen zu sehen waren, weder an der.Hornhaut noch an anderen Teilen.

Von dieser Schnittserie habe ich ein Modell auf 50 fach vergröbsert hergestellt, das in Fig 1 abgebildet ist. Wenn man das Modell etwas näher betrachtet, so kann man die allgemeine Ausbreitung der Gefässe übersichtlich erkennen.

Fig. 1.

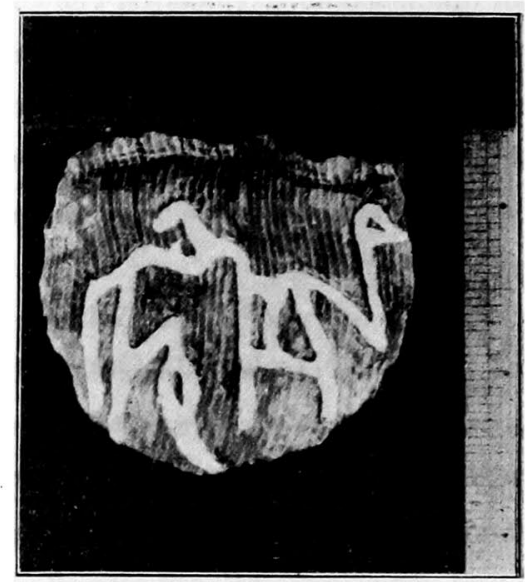

Fig. 1. Siebec Gefïsstämmchen ziehen nach dem Zentrum der Hornhaut hin und bilden miteinander eine ausgedehnte Anastomose. Die-. mictylus pyrrhogaster, von aussen gesehen. 50 fach vergrössert modelliert und auf ca. $\frac{1}{2}$ verkleinert photographiert.

Die Kornealgefässe ziehen in ihren Versorgungsgebieten nach oben hinten von unten vorn. Das untere Zweidrittel der Hornhaut wird von Gefässen versorgt.: Die Randschlingennetze beteiligen sich an der Bildung dieser Gefässe, so dass von den ersteren aus sieben Gefässstämmchen entspringen, von denen fünf den unteren Rand und zwei den vorderen Rand der Hornhaut überschreiten, und diese alle nach dem Zentrum der Hornhaut hinziehen. Die von unten kommenden Gefässe sind meist etwas stärker als die von vorn ausgehenden, wenngleich sie beide ein geringes Kaliber haben. Die Gefässe zeichnen sich durch einé ziemlich ausgedehnte Anastomosenbildung aus. Bevor sie das Zentrum der Hornhaut erreichen, bilden sie miteinander arkadenförmige Anastomosen. Der Bau der 
Kornealgefässe ist ganz ebenso wie bei anderen Kapillaren, indem deren Wand nur aus einer Lage endothelialer Zellen aufgebaut ist. Keine Tunica media und adventitia sind zu sehen.

Fig. 2.

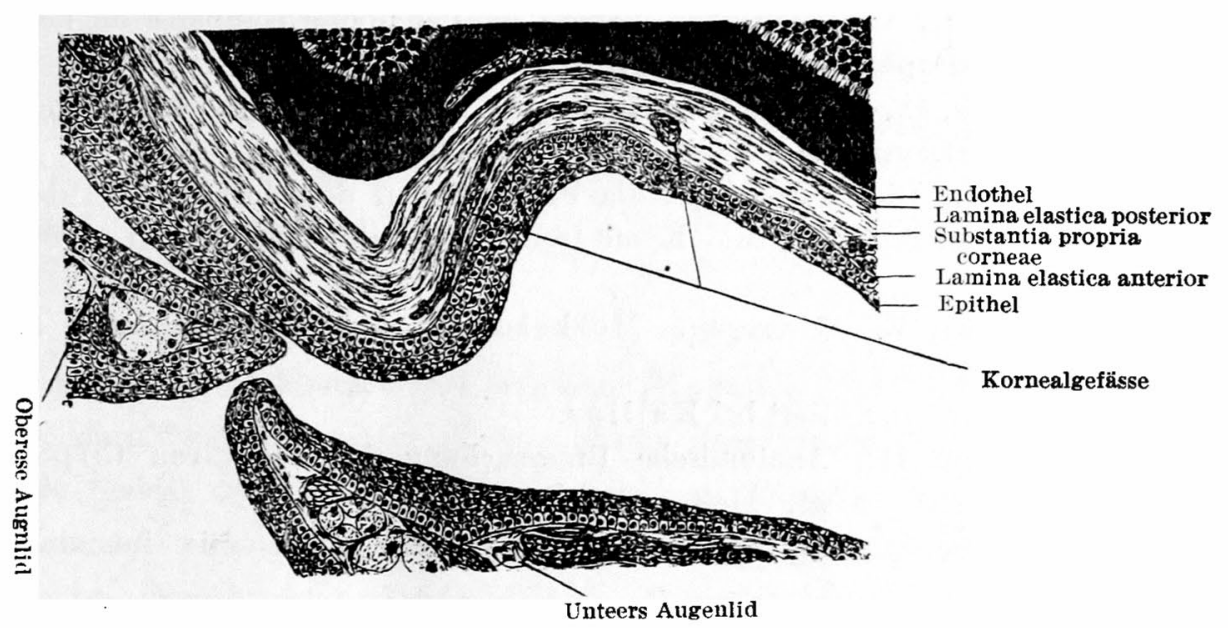

Fig. 2. In der Substantia propria corneae sieht man je einen Längs- und Querschnitt der Kornealgefässe. Diemictylus pyrrhogaster. Objektiv Leitz 3, Okular Leitz 3, Tubuslänge 17,0 mm.

Was die Lage der Gefässe anbelangt, so ist es in meinem Fall etwas anders als bei Lauber (8) und Krause (7). Diese Autoren sind der Meinung, dass bei Cryptobranchus japonicus ein dichter subepithelialer Plexus von Blutgefässen sich in der Hornhaut befindet. Nach $I$ to (5) sind dagegen die Kornealgefässe in der Schicht zwischen dem oberflächlichen einen Drittel und den tiefen zwei Drittel der Substantia propria ausgebildet. Bei meinen Fällen stimmt bei Cryptobranchus der Befund mit der Angabe von Ito vollkommen überein. Bei Diemictylus scheint es mir, dass die Gefässe etwas tiefer gelegen sind als bei Cryptobranchus, beinahe in der mittleren Schicht der Substantia propria.

Das Kornealgefäss von Cryptobranchus japonicus ist, wie schon oben erwähnt, als ein normaler Zustand zu betrachten, während dies bei Diemictylus pyrrhogaster nicht der Fall ist. Doch war bei dem letzteren nirgends ein entzündlicher Prozess zu sehen. Somit will ich diesen sehr seltenen Befund bei Diemictylus als ganz zufällige Erscheinung ansehen. Ich hoffe später bei weiteren Untersuchungen an Urodelen auf die Genese der Kornealgefässe eingehen zu können. 


\section{Literaturverzeichnis.}

1. Berger, E., Beiträge zur Anatomie des Sehorganes der Fische. Morph. Jahrb. Bd. 8. 1883.

2. Berger, E., Anatomie normale et pathologique de l'oeil. 1893.

3. Deyl, J., Contribution à l'étude dé l'anatomie comparée du nerf optique. Bibliographie anatomique: 1898.

4. Hirsch, C., Über die Entwicklung der Hornhautgefässe. Verh. Ges. Deutsch. Naturf. u. Ärzte, Karlsbad. T. 2, Hälfte 2. 1902.

5. Ito, M., Die Gefässe und die Pigmentzellen der Hornhaut bei den japanischen Riesensalamandern. Kyotò-Isen-Koyukwaizasshi. Nr. 51. 1909. (Japanisch).

6. Kallius, E., Schoragan. Merkel-Bonnet, Ergeb. der Anat. u. Entw. Bd. 12. 1902.

7. Krause, R., zitiert bei Kallius.

8. Lauber, H., Anatomische Untersuchung des Auges von Cryptobranchus japonicus. Anat. Hefte. Bd.20. 1903.

9. Motais, M., Poirier et Charpy's Traité d'anatomie humaine. Paris. 1904.

10. Murata, M., Über die Verbreitungsweise der Blutkapillaren in den inneren Organen von Cryptobranchus japonicus. Mitteil. der med. Fakul. der kais. jap. Univ. zu Tokyo. Bd. 9. Heft. 2. 1909.

11. Pütter, A., Organologie des Auges. Graefe-Saemisch, Handb. d. Augenheilkunde. Leipzig. 1908.

12. Rejsek, J., L'histologie de l'oeil de cryptobranchus japonicus. Bibliographie anatomique. 1897. 